\title{
Prognostic significance of aberrantly silenced ANPEP expression in prostate cancer
}

\author{
K D Sørensen ${ }^{\star}, 1$, M O Abildgaard ${ }^{1,2}$, C Haldrup ${ }^{1}$, B P Ulhøi ${ }^{3}, \mathrm{H}$ Kristensen ${ }^{1}$, S Strand ${ }^{1}, \mathrm{C} \mathrm{Parker}^{4}, \mathrm{~S} \mathrm{Høyer}^{3}$, \\ $M$ Borre $^{2}$ and T F Ørntoft ${ }^{\star}, 1$ \\ ${ }^{1}$ Department of Molecular Medicine, Aarhus University Hospital, Brendstrupgaardsvej 100, DK-8200 Aarhus N, Aarhus, Denmark; \\ ${ }^{2}$ Department of Urology, Aarhus University Hospital, Brendstrupgaardsvej 100, DK-8200 Aarhus N, Aarhus, Denmark; Institute of \\ Pathology, Aarhus University Hospital, Nørrebrogade, DK-8000, Aarhus C, Aarhus, Denmark and ${ }^{4}$ Academic Unit of Radiotherapy \\ and Oncology, Institute of Cancer Research and Royal Marsden NHS Foundation Trust, Sutton, SM2 5PT, UK
}

Background: Novel biomarkers for prostate cancer (PC) are urgently needed. This study investigates the expression, epigenetic regulation, and prognostic potential of ANPEP in PC.

Methods: Aminopeptidase N (APN; encoded by ANPEP) expression was analysed by immunohistochemistry using tissue microarrays representing 267 radical prostatectomy (RP) and 111 conservatively treated (CT) PC patients. Clinical end points were recurrence-free survival (RFS) and cancer-specific survival (CSS), respectively. The ANPEP promoter methylation levels were determined by bisulphite sequencing or MethyLight analysis in 278 nonmalignant and PC tissue samples, and in cell lines.

Results: The APN expression was significantly downregulated in PC compared with nonmalignant prostate tissue samples. Aberrant promoter hypermethylation was frequently observed in PC tissue samples, and 5-aza-2'-deoxycytidine induced ANPEP expression in three hypermethylated prostate cell lines, suggesting epigenetic silencing. Negative APN immunoreactivity was significantly associated with short RFS and short CSS in the RP and CT cohort, respectively, independently of routine clinicopathological predictors. Combining APN with a known angiogenesis marker (vascular endothelial growth factor or microvessel density) improved risk prediction significantly in both cohorts.

Conclusion: Our results suggest negative APN immunoreactivity as a new independent adverse prognostic factor for patients with clinically localised PC and, furthermore, that epigenetic mechanisms are involved in silencing of ANPEP in PC.

Improved diagnostic and prognostic biomarkers for prostate cancer (PC) are needed to prevent overtreatment of indolent tumours, and to ensure early detection of aggressive PC that requires treatment. Tissue microarrays (TMAs) have been widely used for discovery of new candidate biomarkers in cancer. Also, there has been increasing focus on the importance in cancer development/progression of epigenetic changes, potentially regulating protein expression. Thus, hypermethylation of promoterassociated $\mathrm{CpG}$ islands is closely linked to gene silencing in cancer.

Alanyl membrane aminopeptidase (ANPEP) encodes a membrane-bound zinc-dependent protease termed aminopeptidase $\mathrm{N}$ (APN; aka neutral aminopeptidase, CD13) that belongs to a group of widely expressed ectopeptidases (Carl-McGrath et al, 2006). The multifunctional APN is responsible for postsecretory processing of, for example, neuropeptides, regulating their access to cellular receptors. The APN is also involved in intracellular cell signalling and seems to play important roles in invasion/metastasis of various malignancies, including PC (Menrad et al, 1993; Ishii et al, 2001; Hashida et al, 2002). Moreover, APN is important for neoangiogenesis (Petrovic et al, 2007).

Although previous reports have shown that APN is downregulated in PC cells compared with nonmalignant prostate epithelial cells (Bogenrieder et al, 1997; Liu et al, 2004; Dall'Era et al, 2007), the mechanism of ANPEP silencing in PC cells has not been previously studied. Furthermore, significant prognostic value has been demonstrated for ANPEP in several malignancies, but

*Correspondence: Dr KD Sørensen or TF Ørntoft;

E-mail: karina.dalsgaard@ki.au.dk or orntoft@ki.au.dk

Revised 26 October 2012; accepted 16 November 2012; published online 15 January 2013

(c) 2013 Cancer Research UK. All rights reserved 0007-0920/13 
remains to be investigated for PC. High expression of APN in cancer cells is associated with poor survival in pancreas (Ikeda et al, 2003) and colon cancer (Hashida et al, 2002). Likewise, high APN expression in tumour-associated endothelial cells predicts poor prognosis in non-small cell lung cancer (Tokuhara et al, 2006). In these cancers, APN expression correlates positively with angiogenesis, as determined by microvessel density (MVD) and/or vascular endothelial growth factor (VEGF) expression, an aspect that has also not been investigated for PC before this study.

In the present study, we investigated the prognostic biomarker potential of ANPEP for PC by immunohistochemical (IHC) analysis of two tissue microarrays representing (1) a PC patient cohort treated by radical prostatectomy $(n=267)$ and $(2)$ a conservatively managed PC patient cohort $(n=111)$. All patients were diagnosed with clinically localised PC. We also examined if $A N P E P$ was hypermethylated in PC, and hence could be a novel epigenetic biomarker candidate.

Our results showed that ANPEP downregulation in PC was frequently associated with aberrant promoter hypermethylation, suggesting that epigenetic mechanisms are involved in silencing of ANPEP in PC cells. In addition, we identified high APN expression on tumour cells as a favourable prognostic factor for clinically localised PC in two treatment regimens: curatively intended radical prostatectomy (RP) and conservative treatment (CT) with no intend to cure.

\section{MATERIALS AND METHODS}

Tissue microarrays. Two TMAs were used, representing RP and CT PC patient cohort, respectively. Tissue specimens were obtained from the Institute of Pathology, Aarhus University Hospital (Aarhus, Denmark). The study was approved by the local Scientific Ethics Committee. For TMA construction, all tissue specimens were evaluated by a pathologist to identify representative areas; Gleason score and tumour stage (RP cohort only) were reassigned according to Epstein et al (2005).

The RP cohort TMA (Heebøll et al, 2008) contained 386 formalin-fixed, paraffin-embedded (FFPE) tissue samples, including $267 \mathrm{RP}$ specimens (Table 1) and several primary tumour specimens from patients with metastatic prostate cancer (MPC), castrate-refractory prostate cancer (CRPC) samples, lymph node metastases (LNMs), cancer-adjacent nonmalignant (AN) prostate tissues, and benign prostatic hyperplasia (BPH) samples (Supplementary Table S1). All RP patients were treated at the Department of Urology, Aarhus University Hospital, from 1998 to 2005.

The CT cohort TMA (Abildgaard et al, 2012) contained tumour specimens from 111 PC patients diagnosed with clinically localised PC from 1979 to 1983 in Aarhus County, Denmark (Table 1). This cohort has been described previously in detail (Borre et al, 1997). All cases were detected incidentally at transurethral resection of the prostate for $\mathrm{BPH}$ or from patients suspected of having PC based on objective findings or symptoms. Prostate-specific antigen (PSA) testing was not used at the time. The CT patients were managed conservatively, that is, followed expectantly and palliated (endocrine treatment; estrogens or orchiectomy) at symptoms only. All patients were followed retrospectively from the time of diagnosis until death (41\% died from PC and 59\% from other causes).

Immunohistochemistry. Citrate buffer was used for epitope demasking. For primary staining, monoclonal mouse anti-human APN/CD13 Ab-3 antibody (clone 38C12; Thermo Fisher Scientific, Slangerup, Denmark) was diluted 1:40 in TBS buffer with 1\% BSA. This antibody has been characterised in several previous studies (Rocken et al, 2005; Terauchi et al, 2007; Mawrin et al, 2010). For secondary staining, we used the anti-mouse EnVision + System
(Dakocytomation, Glostrup, Denmark) with HRP-labelled polymer and DAB solution (Kem-En-Tec, Taastrup, Denmark). Kidney was used as positive control and ovary as negative control for APN (data not shown). As an additional negative control, primary antibody was omitted. Blinded scoring for APN immunoreactivity ( score $=0 \quad$ (negative); score $=1+\quad$ (weak); score $=2+\quad$ (strong intensity)) was performed by a pathologist (BPU) and a medical doctor (MOA) with expertise in prostate histopathology. In cases of disagreement, cores were reassessed to reach a consensus score. The $\kappa$-statistics showed good interobserver agreement ( $\kappa$ index $=0.92 ; P<0.0001)$.

Cell culture. The LNCaP and DU145 prostate adenocarcinoma cells, and BPH1 benign prostatic hyperplasia cells, were cultured as previously described (Abildgaard et al, 2012). Cell lines were treated with 1 to $4 \mu \mathrm{M}$ 5-aza-2'-deoxycytidine (5-aza-dC; Sigma) for $48 \mathrm{~h}$. The media was changed daily and cells were harvested at day 5 for extraction of RNA and DNA.

RNA extraction, cDNA synthesis, and quantitative RT-PCR. See Supplementary Materials and Methods.

Bisulphite sequencing and quantitative methylation-specific PCR analysis. See Supplementary Materials and Methods

Statistical analyses. STATA version 10.1 (StataCorp, College Station TX, USA) was used for all statistical analyses. The $P$-values of $<0.05$ were considered significant. Differences in median expression and methylation levels were analysed by the Mann-Whitney $U$-test. Statistical associations between clinicopathological and IHC data were evaluated by two-sided Fisher's exact and $\left(\chi^{2}\right)$ tests. Statistical correlations between ANPEP/ MYOD1 methylation and APN immunoreactivity were assessed by Spearman's rank test and logistic regression analysis. For the RP cohort, time to PSA recurrence (cutoff $\geqslant 0.2 \mathrm{ng} \mathrm{ml}^{-1}$ ) was selected as the end point. For the CT cohort, cancer-specific (CSS) survival was used as the end point. Survival curves were calculated by the Kaplan-Meier method and evaluated by two-sided log-rank statistics. For recurrence-free survival (RFS) analysis, RP patients were censored at their last tumour-free clinical follow-up visit. Uni- and multivariate Cox regression analyses were used to test the prognostic value of APN immunoreactivity. The proportional hazards assumption was verified by the log-negative-log survival distribution function for all variables.

\section{RESULTS}

APN is downregulated in PC. Using microarray-based transcriptional profiling, we have previously (Sørensen et al, 2009) listed ANPEP as one of the most significantly downregulated genes in PC (Figure 1A). Here, to investigate the expression pattern of APN in detail, we performed IHC analysis of a TMA containing 386 nonmalignant and PC tissue samples (RP cohort TMA; Supplementary Table S1). After exclusion of lost specimens and cores without epithelial cell content, 313 cores (81\%) were scored for APN staining (Figure $1 \mathrm{~B}$ and $\mathrm{C}$ ).

The staining intensities of APN were significantly lower in clinically localised PC (RP specimens) compared with BPH and adjacent nonmalignant (AN) prostate tissue samples (Figure 1B), confirming that APN is downregulated in PC. The vast majority $(>85 \%)$ of benign prostate glands had positive membranous staining (IHC score $=1+$ or $2+$ ) oriented towards the glandular lumen (Figure $1 \mathrm{Ca}$ ), whereas most $(55 \%)$ of the clinically localised PC samples showed no staining (IHC score $=0$; Figure $1 \mathrm{Cb}$ ). The remaining $45 \%$ of clinically localised PC samples were positive (weak or strong intensity) for APN (Figure 1Cc and d), whereas virtually all hormone-naive metastatic PC, castrate-refractory PC, and lymph node metastasis samples were APN negative 
Table 1. Clinicopathological characteristics of PC patient cohorts

\begin{tabular}{|c|c|c|}
\hline & Radical prostatectomy $(n=267)^{a}$ & Conservative treatment $(n=111)^{a}$ \\
\hline Median age, years (range) & $62(46-72)$ & 75 (55-95) \\
\hline \multicolumn{3}{|l|}{ Gleason score, $n$ (\%) } \\
\hline $\begin{array}{l}4-6 \\
7 \\
8-10 \\
\text { Unknown }\end{array}$ & $\begin{array}{l}111(41.6) \\
128(47.9) \\
28(10.5) \\
-\end{array}$ & $\begin{array}{c}37(33.3) \\
20(18.0) \\
53(47.7) \\
1(0.5)\end{array}$ \\
\hline \multicolumn{3}{|l|}{ T stage ${ }^{b}, n(\%)$} \\
\hline $\begin{array}{l}\text { T1 } \\
\text { T2 } \\
\text { T3-4 }\end{array}$ & $\begin{array}{c}- \\
159(59.6) \\
108(40.5)\end{array}$ & $\begin{array}{c}91(82.0) \\
20(18.0) \\
0(0)\end{array}$ \\
\hline
\end{tabular}

PSA at diagnosis, $\boldsymbol{n}(\%)$

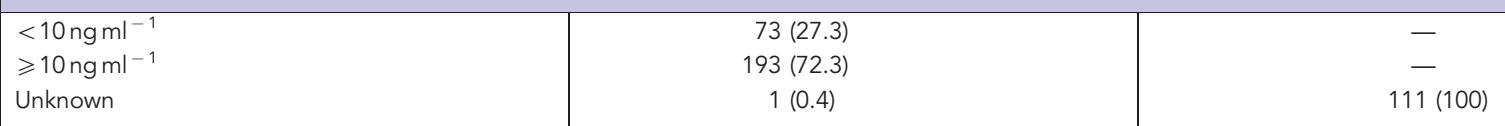

Nodal status, $\boldsymbol{n}(\%)$

\begin{tabular}{|l|r}
\hline pNO & $249(93.3)$ \\
pN1 & $7(2.6)$ \\
Unknown & $11(4.1)$
\end{tabular}

$-$

$-$

$111(100)$

Metastasis status ${ }^{\mathrm{c}}, \mathbf{n}(\%)$

\begin{tabular}{c|c} 
MO & $267(100)$ \\
\hline M1
\end{tabular}

M1

$0(0)$

$111(100)$

$0(0)$

Surgical margin status, $\boldsymbol{n}(\%)$

\begin{tabular}{l|c} 
Negative & $185(69.3)$ \\
Positive & $78(29.2)$ \\
Unknown & $4(1.5)$
\end{tabular}

Early endocrine treatment, $\boldsymbol{n}(\%)$

\begin{tabular}{|r|r} 
No & $267(0)$ \\
\hline Yes & $0(0)$
\end{tabular}

\begin{tabular}{l|r} 
Yes & $0(0)$
\end{tabular}

$68(61.3)$

$43(38.7)$

\section{APN IHC, $n(\%)$}

Score 0

Score $1+$

Score $2+$

Not determined

Median follow-up time, months (range)

$130(48.7)$
$79(29.6)$
$27(10.1)$
$31(11.6)$
$53(1-131)$

NA

Abbreviations: $\mathrm{APN}=$ aminopeptidase $\mathrm{N} ; \mathrm{IHC}=$ immunohistochemistry; $\mathrm{NA}=$ not applicable; $\mathrm{PC}=$ prostate cancer; PSA=prostate-specific antigen.

${ }^{a}$ Both cohorts were based on all consecutive patients visiting the clinic within a specified time period and diagnosed with clinically localised PC, where tissue blocks were available and informed consent obtained (see Materials and Methods for additional information).

bathological for radical prostatectomy (RP) cohort and clinical for conservative treatment (CT) cohort.

${ }^{\mathrm{c}} \mathrm{M} 0$ includes patients without suspicion of metastases at bone scan or X-ray examination as well as patients clinically regarded as having organ-confined disease without objective verification. M1 includes patients with metastases verified by bone scan or X-ray examination as well as patients with manifest clinical symptoms of metastases but without objective verification.

(Figure 1B), indicating that APN is significantly downregulated in metastatic compared with localised disease.

Hypermethylation of ANPEP in PC. We used genomic bisulphite sequencing to investigate if downregulation of $A N P E P$ in PC was associated with hypermethylation of its promoter-associated $\mathrm{CpG}$ island (CGI; Figure 2A). ANPEP was significantly hypermethylated in cancer compared with nonmalignant prostate tissue samples $(P=0.0023$; Mann-Whitney $U$-test $)$ in a clinical sample set consisting of 10 localised PC (LPC), 10 metastatic PC (MPC), and 10 nonmalignant prostate tissue samples (5 AN and $5 \mathrm{BPH}$; Figure $2 \mathrm{~B}$ and Supplementary Figure $\mathrm{S} 1$ ). There was no significant difference in ANPEP methylation levels between BPH and AN prostate tissue samples $(P=0.29)$, nor between LPC and MPC $(P=0.53)$. A single $\mathrm{BPH}$ sample had high-density methylation (70\%) and originated from a patient diagnosed with PC after 2 years. These results suggest that hypermethylation may be a frequent mechanism for silencing of APN in PC.

For validation, we designed a quantitative methylation-specific PCR assay (MethyLight; Figure 2A) and measured ANPEP promoter methylation in 248 nonmalignant and $\mathrm{PC}$ tissue samples also analysed on the RP cohort TMA. A region of MYOD1 not containing $\mathrm{CpG}$ sites was used for normalisation (represents total input of bisulphite converted DNA). Methylation of ANPEP/ MYOD1 was significantly higher in cancer (LPC, MPC, and CRPC) compared with nonmalignant $(\mathrm{AN}$ and $\mathrm{BPH})$ prostate tissue 
A

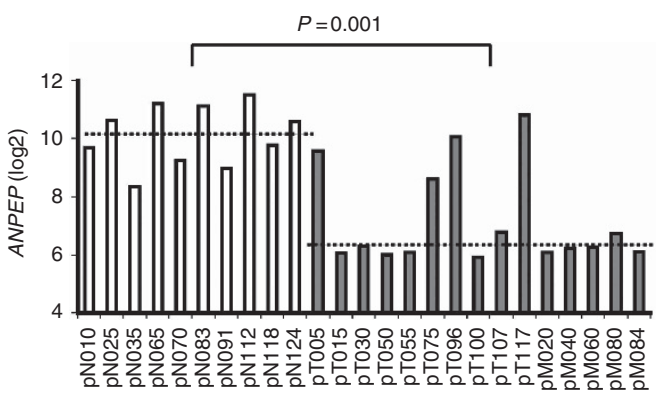

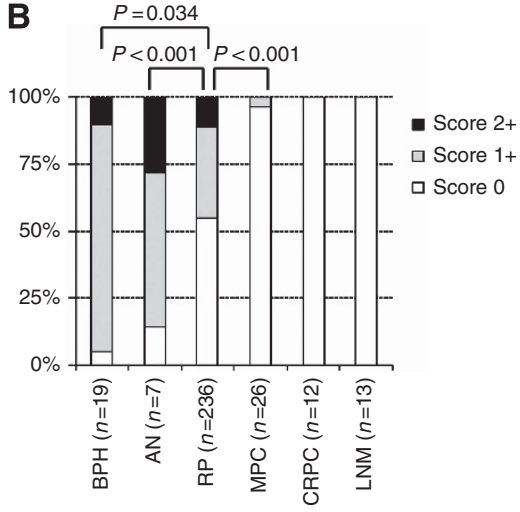

C
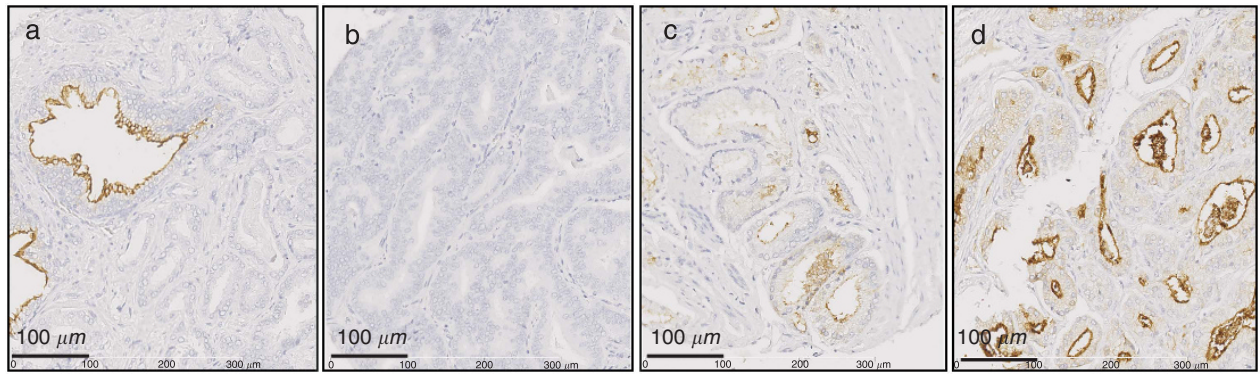

Figure 1. (A) The expression of ANPEP in 10 adjacent nonmalignant prostate (white bars) and 15 PC tissue samples (grey bars) determined by Affymetrix (Santa Clara, CA, USA) Exon Array analysis (data from Thorsen et al, 2008). Horizontal dotted lines indicate median expression in each group. The P-value for Mann-Whitney U-test is given. (B) Distribution of APN immunoreactivity scores relative to tissue specimen types. Number of samples in each group is given in brackets. $\mathrm{BPH}=$ benign prostatic hyperplasia; $\mathrm{AN}=$ adjacent nonmalignant prostate; $\mathrm{RP}=$ radical prostatectomy specimen from patient with clinically localised PC; MPC = primary tumour from patient with hormone-naive metastatic PC; $\mathrm{CRPC}=$ primary tumour from patient with castrate-refractory prostate cancer; $L N M=$ lymph node metastasis. The $P$-values for Fisher's exact tests are given. (C) Representative images of nonmalignant prostate gland (a) and PC (b-d) tissue specimens stained with monoclonal anti-APN antibody. Size bars, $100 \mu \mathrm{m}$. (Ca) Nonmalignant prostate tissue sample with strong intensity APN staining (score $=2+$ ). (Cb) Prostate cancer (PC) tissue sample negative for APN (score $=0)$. $\left(\mathbf{C}_{c}\right)$ Prostate cancer tissue sample showing weak APN staining $($ score $=1+)$. $(\mathbf{C d})$ Prostate cancer tissue sample with strong intensity APN staining (score $=2+$ ).

samples $(P=0.0025$; Mann-Whitney $U$-test $)$, but not significantly different between $\mathrm{AN}$ and $\mathrm{BPH}(P=0.14)$, LPC and MPC $(P=0.19)$, or MPC and CRPC $(P=0.95$; Figure $2 \mathrm{C})$. There was a significant inverse correlation between APN immunoreactivity and ANPEP/MYOD1 methylation levels in this sample set (logistic regression $\mathrm{OR}=0.016, P<0.001 ;$ Spearman $r h o=-0.36$, $P<0.001$ ), indicating that APN downregulation in PC is associated with hypermethylation (Supplementary Figure S2A). Together, our results from two independent patient sample sets (Figure 2B and $\mathrm{C}$ ) indicate that $A N P E P$ is a frequent target of aberrant promoter hypermethylated in PC.

In further support of epigenetic mechanisms controlling ANPEP gene activity in at least some PC cells, treatment with DNA methylation inhibitor 5-aza-dC induced ANPEP transcription significantly in the two hypermethylated PC cell lines LNCaP (10 to 39 times) and DU145 (8 to 17 times) as well as in the hypermethylated BPH1 cell line with undetectable endogenous expression (Figure 2D). Drug-induced gene activation was associated with promoter demethylation in all cell lines (Figure 2D).

In clinical samples, ANPEP/MYOD1 methylation was significantly higher in pT3 vs pT2 tumours and in pN1 vs pN0 tumours $(P=0.0051$ and $P=0.039$, respectively; Mann-Whitney $U$-test; Supplementary Figure S2B). There was no significant correlation with age at diagnosis, PSA, or Gleason score (data not shown). Although low ANPEP/MYOD1 methylation seemed moderately associated with RFS in the RP cohort, the trend was not significant (Supplementary Figure S3). This may suggest that in addition to promoter hypermethylation other mechanisms contribute to the regulation of $A N P E P$ in PC.

Association between APN immunoreactivity, clinicopathological parameters, and angiogenesis markers. Possible correlations between APN expression in PC and routine clinical parameters were analysed for the RP cohort $(n=267)$ and for the CT cohort $(n=111)$ by IHC using tissue microarrays. A total of 236 out of 267 (88\%) and 95 out of $111(86 \%)$ cores, respectively, were assessed for APN expression after exclusion of lost specimens and cores without tumour cell content (Supplementary Table S2). Because of few patients with IHC score $2+$, all cases positive for APN (IHC scores $1+$ and $2+$ ) were merged into a single group. Loss of APN seemed weakly associated with characteristics of aggressive tumours, that is, high Gleason score in both cohorts and advanced pT stage in the RP cohort (Supplementary Table S3), although these trends were only borderline significant. To investigate possible correlations between APN expression and tumour angiogenesis, we used existing VEGF IHC and MVD (CT cohort only) data from these cohorts (Borre et al, 1998; Vergis et al, 2008). There was a significant inverse correlation between APN and VEGF immunoreactivity in the CT cohort $(P=0.018$; Fisher's exact test), but no other significant correlations were observed (Supplementary Table S3). These results for PC are in contrast to reports from other solid tumours, where high APN expression correlates positively with angiogenesis (Hashida et al, 2002; Ikeda et al, 2003; Tokuhara et al, 2006). 
A
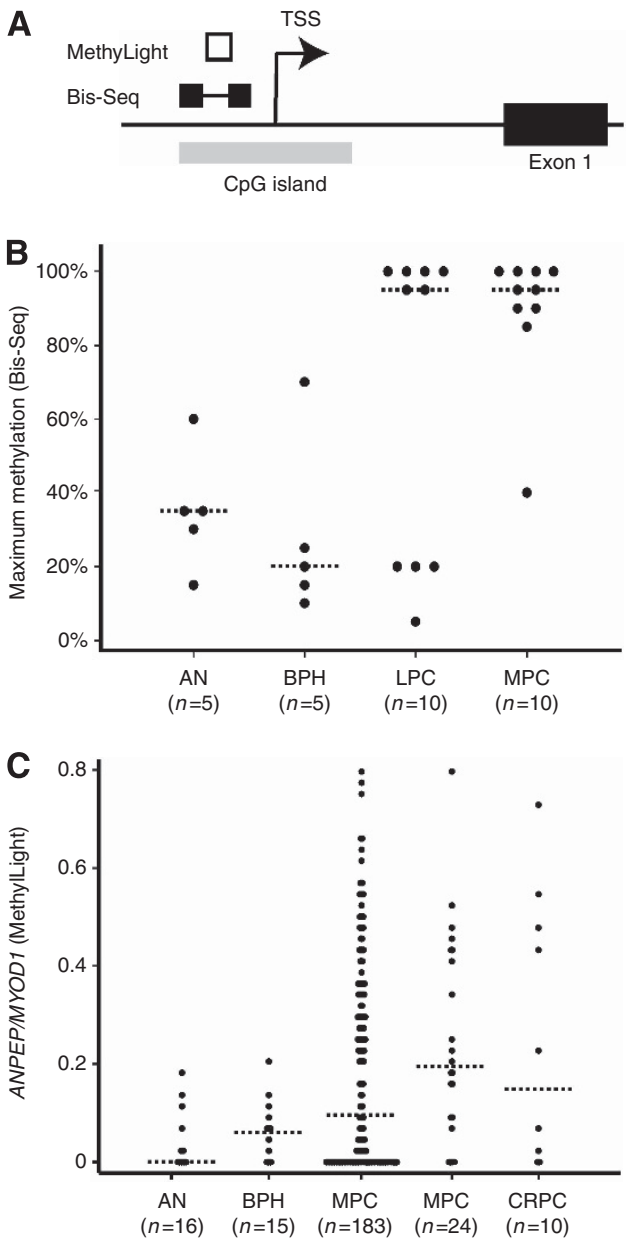

D
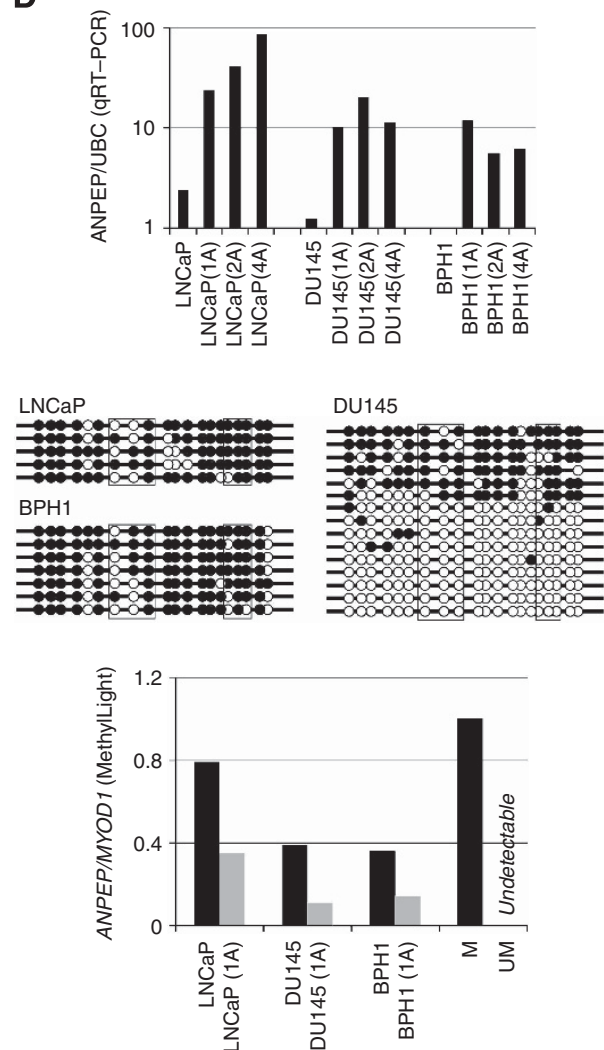

Figure 2. (A) Structure of ANPEP genomic locus. Location of the promoter-associated CpG island is shown by a grey box. DNA regions analysed by bisulphite sequencing (Bis-Seq) and quantitative methylation-specific PCR (MethyLight) are indicated above (dumbbell and white box, respectively). TSS, transcription start site within CPG island. The figure is not to scale. (B) Dot plot of ANPEP promoter methylation in a small clinical sample set analysed by bisulphite sequencing. Percent methylated CpG sites/total number of CpG sites in the most heavily methylated clone is given for each sample (see also Supplementary Figure S1). (C) Dot plot of relative ANPEP promoter methylation (normalised to MYOD1) in a second and larger clinical sample set, determined by MethyLight analysis. (B and C) Horizontal dashed lines indicate median methylation for each group. Sample numbers in each group are given in brackets. (D) Normalised ANPEP expression in three prostate cell lines treated with $1 \mu \mathrm{M}$ (1A), $2 \mu \mathrm{M}(2 \mathrm{~A})$, or $4 \mu \mathrm{M}(4 \mathrm{~A}) 5$-aza-2'-deoxycytidine or untreated is shown at the top. The expression of ANPEP was determined by qRT-PCR using UBC for normalisation, and further normalised to ANPEP/UBC expression in a pool of four adjacent nonmalignant prostate tissue samples (arbitrarily set to 100; not shown). The expression of ANPEP was induced by 5-aza-2'-deoxycytidine in all cell lines. Bisulphite sequencing results for ANPEP in prostate cell lines is shown below. Open and closed circles indicate unmethylated and methylated CpGs, respectively. Each row represents one clone. The CpG sites interrogated by MethyLight analysis are highlighted by black boxes. The promoter methylation levels of ANPEP in treated and untreated cell lines, methylated (M) and unmethylated (UM) control DNA samples, determined by MethyLight analysis, is shown at the bottom.

Univariate and multivariate survival analyses. To assess the possible prognostic value of APN, we performed PSA-based RFS analysis for the RP cohort. By univariate analysis, negative APN immunoreactivity was significantly associated with short RFS (Figure 3A; $P=0.0019$ ). Other variables associated with short RFS in the RP cohort were high preoperative PSA, high Gleason score, advanced $\mathrm{T}$ stage, positive lymph nodes, positive surgical margins, and high VEGF staining (Table 2). Except for nodal status and $\mathrm{T}$ stage, all variables remained significant in a multivariate model (Table 2), indicating that APN immunoreactivity is a significant independent prognostic factor for PC patients with clinically localised disease. Risk stratification (low/intermediate/high) in the RP cohort was significantly improved by combining APN and VEGF in a simple two-marker model (Figure 3B).

Using the same cut-off (negative $v s$ positive immunoreactivity), the prognostic value of APN for clinically localised PC was successfully validated in the independent CT cohort (Figure 3C and $\mathrm{D}$ and Table 3 ). In this cohort, negative APN was significantly associated with short CSS by univariate (Figure 3C; $P=0.0065$ ) as well as multivariate analyses (Table 3). High Gleason score and high MVD were also significantly associated with short CSS in the CT cohort by uni- and multivariate analyses (Table 3). VEGF immunoreactivity was significant only by univariate analysis in the CT cohort. Prediction of CSS was significantly improved by combining APN immunoreactivity and MVD (Figure 3D). There was no significant association between APN staining and overall survival in the CT cohort (data not shown).

\section{DISCUSSION}

In summary, our results based on two distinct PC patient cohorts indicate that negative APN immunoreactivity is a significant 

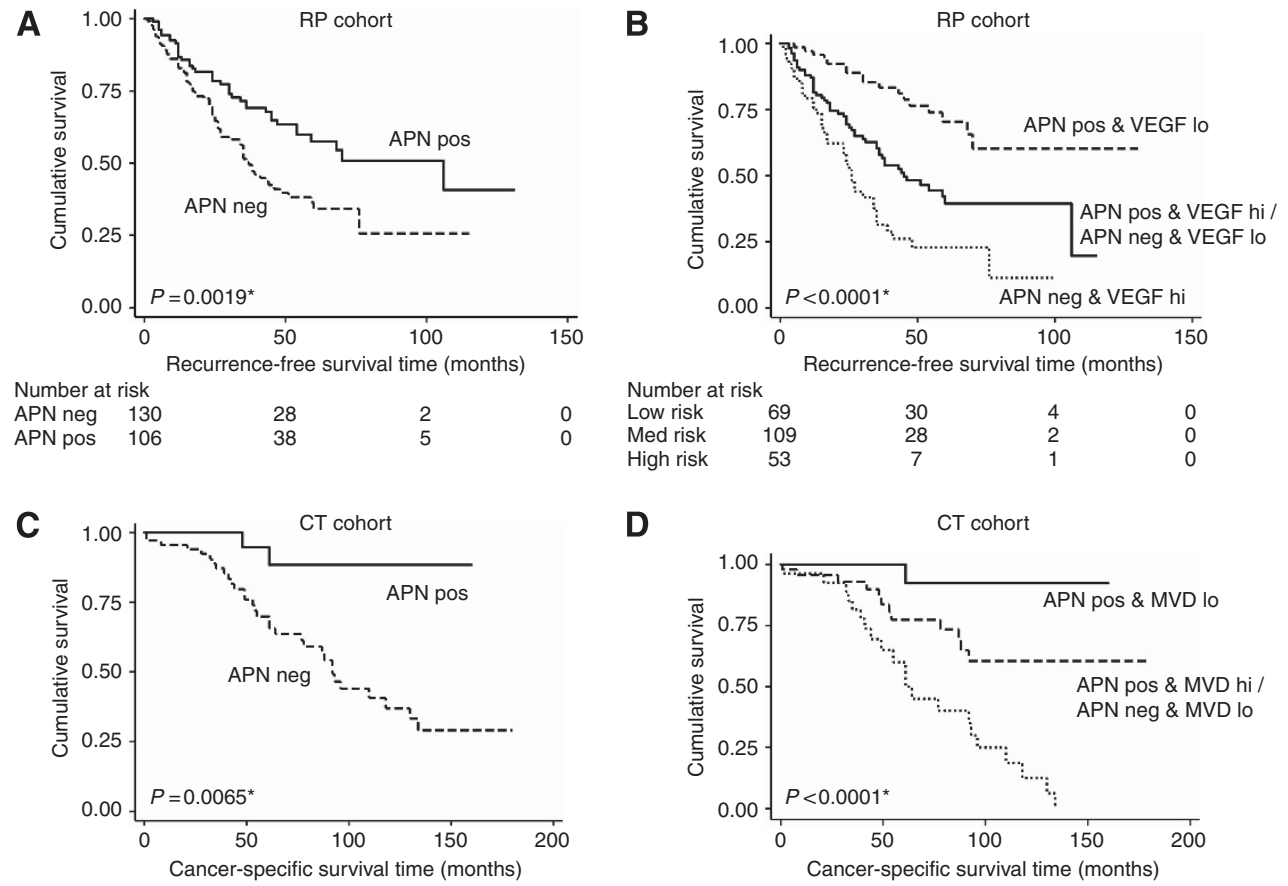

D

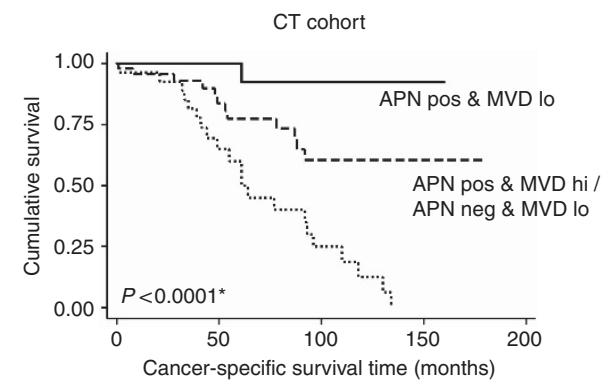

\begin{tabular}{|c|c|c|c|c|}
\hline & & & & \\
\hline APN neg & 69 & 40 & 16 & 5 \\
\hline APN pos & 26 & 17 & 5 & 1 \\
\hline
\end{tabular}

$\begin{array}{lccccc}\text { Number at risk } & & & & \\ \text { Low risk } & 20 & 15 & 4 & 1 & 0 \\ \text { Med risk } & 48 & 27 & 12 & 5 & 0 \\ \text { High risk } & 27 & 15 & 5 & 0 & 0\end{array}$

Figure 3. Kaplan-Meier plots of RFS for the RP cohort ( $\mathbf{A}$ and $\mathbf{B}$ ) and CSS for the CT cohort (C and $\mathbf{D})$. The $P$-values for two-sided log-rank statistics are given, comparing patients with negative $(\mathrm{IHC}$ score $=0)$ or positive $(\mathrm{IHC}$ score $=1+$ or $2+)$ APN immunoreactivity $(\mathbf{A}, \mathbf{C})$, as well as patients with positive/negative APN staining in combination with low/high VEGF staining (score 0-2 vs score 3-5) in the RP cohort (B), or low/high microvessel density (MVD; dichotomised at median) in the CT cohort (D). * Significant $P$-values.

Table 2. Cox regression analyses for RP cohort (recurrence-free survival)

\begin{tabular}{|c|c|c|c|c|c|c|}
\hline \multirow{2}{*}{$\begin{array}{l}\text { RP cohort }(n=267) \\
\text { Variable (characteristics) }\end{array}$} & \multicolumn{2}{|c|}{ Univariate } & \multicolumn{2}{|c|}{ Multivariate (global model) } & \multicolumn{2}{|c|}{ Multivariate (final model) } \\
\hline & $\mathrm{HR}(95 \% \mathrm{Cl})$ & $\boldsymbol{P}$-value & $\mathrm{HR}(95 \% \mathrm{Cl})$ & $\boldsymbol{P}$-value & $\mathrm{HR}(95 \% \mathrm{Cl})$ & $P$-value \\
\hline Age at diagnosis ( $\leqslant 62$ vs $>62$ years) & $1.05(0.73-1.50)$ & 0.80 & - & - & - & - \\
\hline Preoperative PSA (continuous) & $1.05(1.04-1.07)$ & $<0.001$ & $1.04(1.03-1.06)$ & $<0.001$ & $1.04(1.03-1.06)$ & $<0.001$ \\
\hline Gleason score (5-6 vs 7-10) & $2.81(1.83-4.31)$ & $<0.001$ & $1.60(0.99-2.60)$ & 0.056 & $1.72(1.08-2.75)$ & 0.023 \\
\hline Tumour stage (pT2 vs $\mathrm{pT} 3$ ) & $3.35(2.30-4.88)$ & $<0.001$ & $1.54(0.88-2.69)$ & 0.13 & - & - \\
\hline Nodal status ( $\mathrm{pNO}$ vs $\mathrm{pN} 1$ ) & $3.36(1.56-7.24)$ & 0.002 & $1.68(0.71-3.98)$ & 0.24 & - & - \\
\hline Surgical margins (neg vs pos) & $3.05(2.11-4.41)$ & $<0.001$ & $1.77(1.03-3.04)$ & 0.038 & $2.38(1.61-3.52)$ & $<0.001$ \\
\hline VEGF (low vs high) & $2.84(1.97-4.11)$ & $<0.001$ & $2.12(1.41-3.20)$ & $<0.001$ & $2.05(1.38-3.06)$ & $<0.001$ \\
\hline APN IHC (neg vs pos) & $0.55(0.37-0.80)$ & 0.002 & $0.64(0.42-0.98)$ & 0.039 & $0.54(0.36-0.80)$ & 0.017 \\
\hline
\end{tabular}

independent adverse prognostic factor for patients diagnosed with clinically localised PC. Although further validation is needed, this indicates that a simple IHC test for APN may add significant prognostic value to currently used routine predictors for PC patient outcome.

Furthermore, we identified ANPEP as a novel common target of aberrant promoter hypermethylated in PC. Hypermethylation correlated inversely with APN expression, indicating that ANPEP is epigenetically silenced in PC cells. To our knowledge, this is the first report of aberrant ANPEP hypermethylation in PC. As further experimental evidence for epigenetic regulation of ANPEP expression, 5-aza-dC treatment resulted in promoter demethylation and transcriptional reactivation in three hypermethylated prostate cell lines. Recently, hypermethylation and downregulation of ANPEP were reported for some malignant melanoma cells (Wulfanger et al, 2012).

The methylation levels of ANPEP did not have significant prognostic value in the RP cohort (it was borderline significant, but failed to validate in an independent patient cohort (data not shown)). A likely explanation is that other mechanisms, in addition to promoter hypermethylation, contribute to downregulation of ANPEP in PC cells. Absence of essential transcription factor activities may prevent transcription from unmethylated promoters, and the repressive histone mark H3K27me3 can 
Table 3. Cox regression analyses for CT cohort (cancer-specific survival)

\begin{tabular}{|c|c|c|c|c|c|c|}
\hline \multirow{2}{*}{$\begin{array}{l}\text { CT cohort }(n=111) \\
\text { Variable (characteristics) }\end{array}$} & \multicolumn{2}{|c|}{ Univariate } & \multicolumn{2}{|c|}{ Multivariate (global model) } & \multicolumn{2}{|c|}{ Multivariate (final model) } \\
\hline & $\mathrm{HR}(95 \% \mathrm{Cl})$ & $\boldsymbol{P}$-value & $\mathrm{HR}(95 \% \mathrm{Cl})$ & $\boldsymbol{P}$-value & $\mathrm{HR}(95 \% \mathrm{Cl})$ & $P$-value \\
\hline Age at diagnosis ( $\leqslant 74$ vs $>74$ years) & $0.94(0.53-1.68)$ & 0.84 & - & - & - & - \\
\hline Gleason score (5-6 vs 7-10) & $4.08(1.72-9.69)$ & 0.001 & $3.16(1.08-9.27)$ & 0.036 & $3.48(1.20-10.1)$ & 0.021 \\
\hline Endocrine treatment (yes/no) & $0.97(0.54-1.75)$ & 0.93 & - & - & - & - \\
\hline Microvessel density (continuous) & $1.09(1.05-1.14)$ & $<0.001$ & $1.08(1.02-1.13)$ & 0.005 & $1.09(1.03-1.14)$ & 0.001 \\
\hline VEGF (low vs high) & $2.34(1.28-4.27)$ & 0.006 & $1.61(0.76-3.43)$ & 0.213 & - & - \\
\hline APN IHC (neg vs pos) & $0.17(0.04-0.72)$ & 0.017 & $0.27(0.06-1.19)$ & 0.084 & $0.23(0.06-1.00)$ & 0.049 \\
\hline
\end{tabular}

silence genes independently of DNA methylation (Sharma et al, 2010). Future studies should elucidate in more detail the transcriptional regulation of ANPEP in normal and PC cells.

Our TMA analysis results expand and confirm results from previous IHC studies of APN in nonmalignant and PC tissue samples (Bogenrieder et al, 1997; Liu et al, 2004; Dall'Era et al, 2007). Significant correlations between APN protein expression and PC prognosis have not been demonstrated before this report, possibly because of insufficient sample sizes in the former studies. Recently, low ANPEP transcript levels were associated with PSA recurrence after RP in a set of 29 PC patient samples, but no independent validation was provided (Larkin et al, 2012). Hence, the present study is the first to conclusively demonstrate a significant independent prognostic value of ANPEP expression in PC.

The anti-APN antibody (38C12) used here does not work on cryosections, whereas the antibodies (WM15 and F23) used in previous reports (Bogenrieder et al, 1997; Liu et al, 2004; Dall'Era et al, 2007) do not work with FFPE samples. Furthermore, the $38 \mathrm{C} 12$ antibody did not work for western blotting in our hands (data not shown), as also experienced by others (Mawrin et al, 2010). In summary, however, highly similar results have now been obtained by IHC analysis with three different monoclonal antiAPN antibodies, strongly supporting the validity of our findings. Reduced APN immunoreactivity in PC is in agreement with transcriptional downregulation of $A N P E P$, as reported in microarray studies (Liu et al, 2006; Tamura et al, 2007; Thorsen et al, 2008). Consistent with previous reports (Bogenrieder et al, 1997; Liu et al, 2004; Dall'Era et al, 2007), we detected APN staining of the luminal cell membrane in nonmalignant secretory epithelial cells, whereas basal cells were APN negative. Loss of APN may be associated with dedifferentiation of normal prostate epithelial cells, potentially promoting prostate carcinogenesis. Notably, ANPEP is downregulated in colon epithelial cell subpopulations enriched for intestinal stem cells (Jung et al, 2011).

Downregulation of APN in metastatic compared with localised PC may suggest a potential role for APN also in tumour progression. Although APN stimulates invasion of the PC3 prostate cancer cell line (Ishii et al, 2001), which shares characteristics of small cell neuroendocrine PC (Tai et al, 2011), the exact function of APN in normal and prostate adenocarcinoma cells remains unclear. In fact, the observed downregulation of APN in clinical PC and its association with poor prognosis may suggest that APN plays a predominantly tumour-suppressive role in clinical prostate adenocarcinoma. Reports for several other cancer types indicate that APN functions in a highly cell type- and context-specific manner. Thus, low APN expression is associated with aggressive disease in meningioma and gastric cancer (Kawamura et al, 2007; Mawrin et al, 2010), whereas high expression is an adverse prognostic factor for lung, pancreas and colon cancer (Hashida et al, 2002; Ikeda et al, 2003; Murakami et al, 2005; Tokuhara et al, 2006). Future studies should resolve the biological function(s) of APN in prostate cells and determine whether downregulation of APN is a driver or passenger aberration in PC.

Overall, our results suggest that an IHC test for APN in prostate tumour specimens may add significant prognostic value at the time of diagnosis, and thereby potentially could aid in treatment decision making for clinically localised PC. The fact that a simple two-marker test (APN and VEGF, or APN and MVD) had even stronger prognostic power in the RP and CT cohorts suggest that a combined test may have superior clinical utility. The results presented here confirm our previous findings that (1) MVD, but not VEGF, has significant independent prognostic value for CSS in the CT cohort (Borre et al, 1998, 2000) and (2) VEGF is an independent predictor of RFS in the RP cohort (Vergis et al, 2008). The data for MVD were unavailable for the RP cohort. Several conflicting reports exist regarding the prognostic power of VEGF and MVD for PC (Green et al, 2007; Revelos et al, 2007; Mucci et al, 2009; Boxler et al, 2010), and it is possible that other markers of angiogenesis can improve PC risk stratification beyond the findings in this study.

An IHC test for Nestin/Ki-67, reflecting immature vascular proliferation, was recently found superior to MVD and VEGF for outcome prediction in PC patients (Gravdal et al, 2009). Another study highlighted vascular morphology (size and irregularity of tumour-associated vessels) as a more accurate predictor of PCspecific death than MVD (Mucci et al, 2009). Future studies should investigate if these or other emerging markers of angiogenesis will improve risk stratification further if combined with APN. Finally, it is important to note that our approach used prostatectomy specimens for the RP cohort and TURP specimens for the CT cohort. It will be interesting to investigate if the prognostic value of APN alone or in combination with a marker of angiogenesis can be transferred to prostate biopsies at the time of diagnosis.

In conclusion, our findings suggest that a simple and inexpensive IHC analysis for APN combined with a marker of angiogenesis may be used together with routine prognostic variables ( $T$ stage, PSA, Gleason score) to stratify patients into low-, intermediate-, and high-risk groups. Notably, the combined test (APN plus MVD) identified a significant subpopulation (18\%) of CT patients with very low risk $(<10 \%)$ of dying from PC (see Figure $3 \mathrm{D}$ ). Although further validation is needed, these results suggest that an APN-based test may be particularly useful for guiding the selection of PC patients for watchful waiting or active surveillance. These are becoming preferred treatment options for the increasing number of patients diagnosed with low-risk clinically localised PC as a result of widespread PSA testing. 


\section{ACKNOWLEDGEMENTS}

We thank Dr Niels Tørring for advice on IHC analysis, and Anne Slotsdal, Pamela Celis, Maria Mark, Gitte Høj, Mette Rasmussen, Conni Sørensen, and Susanne Skou for excellent technical assistance. This work was supported by The Lundbeck Foundation, The John and Birthe Meyer Foundation, The Danish Cancer Society, and The Danish Council for Strategic Research. The Danish Cancer Biobank (DCB) is acknowledged for biological material.

\section{CONFLICT OF INTEREST}

The authors declare no conflict of interest.

\section{REFERENCES}

Abildgaard MO, Borre M, Mortensen MM, Ulhøi BP, Tørring N, Wild P, Kristensen H, Mansilla F, Ottosen PD, Dyrskjøt L, Ørntoft TF, Sørensen KD (2012) Downregulation of zinc finger protein 132 in prostate cancer is associated with aberrant promoter hypermethylation and poor prognosis. Int J Cancer 130(4): 885-895.

Bogenrieder T, Finstad CL, Freeman RH, Papandreou CN, Scher HI, Albino AP, Reuter VE, Nanus DM (1997) Expression and localization of aminopeptidase $\mathrm{A}$, aminopeptidase $\mathrm{N}$, and dipeptidyl peptidase IV in benign and malignant human prostate tissue. Prostate 33(4): 225-232.

Borre M, Nerstrøm B, Overgaard J (1997) The natural history of prostate carcinoma based on a Danish population treated with no intent to cure. Cancer 80(5): 917-928.

Borre M, Nerstrøm B, Overgaard J (2000) Association between immunohistochemical expression of vascular endothelial growth factor (VEGF), VEGFexpressing neuroendocrine-differentiated tumor cells, and outcome in prostate cancer patients subjected to watchful waiting. Clin Cancer Res 6(5): 1882-1890.

Borre M, Offersen BV, Nerstrøm B, Overgaard J (1998) Microvessel density predicts survival in prostate cancer patients subjected to watchful waiting. Br J Cancer 78(7): 940-944.

Boxler S, Djonov V, Kessler TM, Hlushchuk R, Bachmann LM, Held U, Markwalder R, Thalmann GN (2010) Matrix metalloproteinases and angiogenic factors: predictors of survival after radical prostatectomy for clinically organ-confined prostate cancer? Am J Pathol 177(5): 2216-2224.

Carl-McGrath S, Lendeckel U, Ebert M, Rocken C (2006) Ectopeptidases in tumour biology: a review. Histol Histopathol 21(12): 1339-1353.

Dall'Era MA, True LD, Siegel AF, Porter MP, Sherertz TM, Liu AY (2007) Differential expression of CD10 in prostate cancer and its clinical implication. BMC Urol 7: 3.

Epstein JI, Allsbrook Jr. WC, Amin MB, Egevad LL (2005) The 2005 International Society of Urological Pathology (ISUP) Consensus Conference on Gleason Grading of Prostatic Carcinoma. Am J Surg Pathol 29(9): 1228-1242.

Gravdal K, Halvorsen OJ, Haukaas SA, Akslen LA (2009) Proliferation of immature tumor vessels is a novel marker of clinical progression in prostate cancer. Cancer Res 69(11): 4708-4715.

Green MM, Hiley CT, Shanks JH, Bottomley IC, West CM, Cowan RA, Stratford IJ (2007) Expression of vascular endothelial growth factor (VEGF) in locally invasive prostate cancer is prognostic for radiotherapy outcome. Int J Radiat Oncol Biol Phys 67(1): 84-90.

Hashida H, Takabayashi A, Kanai M, Adachi M, Kondo K, Kohno N, Yamaoka Y, Miyake M (2002) Aminopeptidase N is involved in cell motility and angiogenesis: its clinical significance in human colon cancer. Gastroenterology 122(2): 376-386.

Heebøll S, Borre M, Ottosen PD, Andersen CL, Mansilla F, Dyrskjøt L, Ørntoft TF, Tørring N (2008) SMARCC1 expression is upregulated in prostate cancer and positively correlated with tumour recurrence and dedifferentiation. Histol Histopathol 23(9): 1069-1076.

Ikeda N, Nakajima Y, Tokuhara T, Hattori N, Sho M, Kanehiro H, Miyake M (2003) Clinical significance of aminopeptidase N/CD13 expression in human pancreatic carcinoma. Clin Cancer Res 9(4): 1503-1508.
Ishii K, Usui S, Sugimura Y, Yoshida S, Hioki T, Tatematsu M, Yamamoto H, Hirano K (2001) Aminopeptidase N regulated by zinc in human prostate participates in tumor cell invasion. Int J Cancer 92(1): $49-54$.

Jung P, Sato T, Merlos-Suarez A, Barriga FM, Iglesias M, Rossell D, Auer H, Gallardo M, Blasco MA, Sancho E, Clevers H, Batlle E (2011) Isolation and in vitro expansion of human colonic stem cells. Nat Med 17(10): 1225-1227.

Kawamura J, Shimada Y, Kitaichi H, Komoto I, Hashimoto Y, Kaganoi J, Miyake M, Yamasaki S, Kondo K, Imamura M (2007) Clinicopathological significance of aminopeptidase N/CD13 expression in human gastric carcinoma. Hepatogastroenterology 54(73): 36-40.

Larkin SE, Holmes S, Cree IA, Walker T, Basketter V, Bickers B, Harris S, Garbis SD, Townsend PA, Aukim-Hastie C (2012) Identification of markers of prostate cancer progression using candidate gene expression. Br J Cancer 106(1): 157-165.

Liu AY, Roudier MP, True LD (2004) Heterogeneity in primary and metastatic prostate cancer as defined by cell surface $\mathrm{CD}$ profile. $\mathrm{Am} J$ Pathol 165(5): 1543-1556.

Liu P, Ramachandran S, Ali Seyed M, Scharer CD, Laycock N, Dalton WB, Williams H, Karanam S, Datta MW, Jaye DL, Moreno CS (2006) Sexdetermining region $\mathrm{Y}$ box 4 is a transforming oncogene in human prostate cancer cells. Cancer Res 66(8): 4011-4019.

Mawrin C, Wolke C, Haase D, Kruger S, Firsching R, Keilhoff G, Paulus W, Gutmann DH, Lal A, Lendeckel U (2010) Reduced activity of CD13/ aminopeptidase N (APN) in aggressive meningiomas is associated with increased levels of SPARC. Brain Pathol 20(1): 200-210.

Menrad A, Speicher D, Wacker J, Herlyn M (1993) Biochemical and functional characterization of aminopeptidase $\mathrm{N}$ expressed by human melanoma cells. Cancer Res 53(6): 1450-1455.

Mucci LA, Powolny A, Giovannucci E, Liao Z, Kenfield SA, Shen R, Stampfer MJ, Clinton SK (2009) Prospective study of prostate tumor angiogenesis and cancer-specific mortality in the health professionals follow-up study. J Clin Oncol 27(33): 5627-5633.

Murakami H, Yokoyama A, Kondo K, Nakanishi S, Kohno N, Miyake M (2005) Circulating aminopeptidase N/CD13 is an independent prognostic factor in patients with non-small cell lung cancer. Clin Cancer Res 11(24 Pt 1): 8674-8679.

Petrovic N, Schacke W, Gahagan JR, O'Conor CA, Winnicka B, Conway RE, Mina-Osorio P, Shapiro LH (2007) CD13/APN regulates endothelial invasion and filopodia formation. Blood 110(1): $142-150$.

Revelos K, Petraki C, Scorilas A, Stefanakis S, Malovrouvas D, Alevizopoulos N, Kanellis G, Halapas A, Koutsilieris M (2007) Correlation of androgen receptor status, neuroendocrine differentiation and angiogenesis with time-to-biochemical failure after radical prostatectomy in clinically localized prostate cancer. Anticancer Res 27(5B): 3651-3660.

Rocken C, Licht J, Roessner A, Carl-McGrath S (2005) Canalicular immunostaining of aminopeptidase $\mathrm{N}(\mathrm{CD} 13)$ as a diagnostic marker for hepatocellular carcinoma. J Clin Pathol 58(10): 1069-1075.

Sharma S, Kelly TK, Jones PA (2010) Epigenetics in cancer. Carcinogenesis 31(1): 27-36.

Sørensen KD, Wild PJ, Mortezavi A, Adolf K, Tørring N, Heebøll S, Ulhøi BP, Ottosen P, Sulser T, Hermanns T, Moch H, Borre M, Ørntoft TF, Dyrskjøt L (2009) Genetic and epigenetic SLC18A2 silencing in prostate cancer is an independent adverse predictor of biochemical recurrence after radical prostatectomy. Clin Cancer Res 15(4): $1400-1410$.

Tai S, Sun Y, Squires JM, Zhang H, Oh WK, Liang CZ, Huang J (2011) PC3 is a cell line characteristic of prostatic small cell carcinoma. Prostate 71(15): 1668-1679.

Tamura K, Furihata M, Tsunoda T, Ashida S, Takata R, Obara W, Yoshioka H, Daigo Y, Nasu Y, Kumon H, Konaka H, Namiki M, Tozawa K, Kohri K, Tanji N, Yokoyama M, Shimazui T, Akaza H, Mizutani Y, Miki T, Fujioka T, Shuin T, Nakamura Y, Nakagawa H (2007) Molecular features of hormone-refractory prostate cancer cells by genome-wide gene expression profiles. Cancer Res 67(11): 5117-5125.

Terauchi M, Kajiyama H, Shibata K, Ino K, Nawa A, Mizutani S, Kikkawa F (2007) Inhibition of APN/CD13 leads to suppressed progressive potential in ovarian carcinoma cells. BMC Cancer 7: 140. 
Thorsen K, Sørensen KD, Brems-Eskildsen AS, Modin C, Gaustadnes M, Hein AM, Kruhøffer M, Laurberg S, Borre M, Wang K, Brunak S, Krainer AR, Tørring N, Dyrskjøt L, Andersen CL, Ørntoft TF (2008) Alternative splicing in colon, bladder, and prostate cancer identified by exon array analysis. Mol Cell Proteomics 7(7): 1214-1224.

Tokuhara T, Hattori N, Ishida H, Hirai T, Higashiyama M, Kodama K, Miyake M (2006) Clinical significance of aminopeptidase N in non-small cell lung cancer. Clin Cancer Res 12(13): 3971-3978.

Vergis R, Corbishley CM, Norman AR, Bartlett J, Jhavar S, Borre M, Heeboll S, Horwich A, Huddart R, Khoo V, Eeles R, Cooper C, Sydes M, Dearnaley D, Parker C (2008) Intrinsic markers of tumour hypoxia and angiogenesis in localised prostate cancer and outcome of radical treatment: a retrospective analysis of two randomised radiotherapy trials and one surgical cohort study. Lancet Oncol 9(4): 342-351.

Wulfanger J, Schneider H, Wild P, Ikenberg K, Rodolfo M, Rivoltini L, Meyer S, Riemann D, Seliger B (2012) Promoter methylation of aminopeptidase N/CD13 in malignant melanoma. Carcinogenesis 33(4): 781-790.

This work is published under the standard license to publish agreement. After 12 months the work will become freely available and the license terms will switch to a Creative Commons AttributionNonCommercial-Share Alike 3.0 Unported License.

Supplementary Information accompanies this paper on British Journal of Cancer website (http://www.nature.com/bjc) 